\title{
Hyperon production in near threshold nucleon-nucleon collisions
}

\author{
Radhey Shyam \\ ${ }^{1}$ Saha Institute of Nuclear Physics, Kolkata, India
}

(Dated: May 5, 2018)

\begin{abstract}
We study the mechanism of the associated $\Lambda$-kaon and $\Sigma$-kaon production in nucleon-nucleon collisions over an extended range of near threshold beam energies within an effective Lagrangian model, to understand the new data on $p p \rightarrow p \Lambda K^{+}$and $p p \rightarrow p \Sigma^{0} K^{+}$reactions published recently by the COSY-11 collaboration. In this theory, the hyperon production proceeds via the excitation of $N^{*}(1650), N^{*}(1710)$, and $N^{*}(1720)$ baryonic resonances. Interplay of the relative contributions of various resonances to the cross sections, is discussed as a function of the beam energy over a larger near threshold energy domain. Predictions of our model are given for the total cross sections of $p p \rightarrow p \Sigma^{+} K^{0}, p p \rightarrow n \Sigma^{+} K^{+}$, and $p n \rightarrow n \Lambda K^{+}$reactions.

PACS numbers: 13.60.Le, 13.75.Cs, 11.80. $-m, 12.40 . V v$
\end{abstract}


Dedicated experiments performed recently at the Cooler Synchrotron (COSY) facility at the Forschungszentrum, Jülich, have led to the accumulation of high quality data on associated hyperon production in close-to-threshold proton-proton $(p p)$ collisions [1, 2, 3, 4, 15]. Strangeness production reactions are expected to provide information on the manifestation of quantum chromodynamics (QCD) in the non-perturbative regime of energies larger than those of the low energy pion physics where the low energy theorem and partial conservation of axial current (PCAC) constraints provide a useful insight into the relevant physics [6]. The strangeness quantum number introduced by this reaction leads to new degrees of freedom in this domain which are likely to probe the admixture of $\bar{s} s$ quark pairs in the nucleon wave function 7]. At the near threshold beam energies, the final state interaction (FSI) effects among the outgoing particles are significant. Therefore, the new set of data are expected to probe also the hyperon-nucleon and hyperon-strange meson interactions (see, e.g., Refs. [8, 9, 10]).

A very interesting result of the studies performed by the COSY-11 collaboration is that the ratio $(R)$ of the total cross sections for the $p p \rightarrow p \Lambda K^{+}$and $p p \rightarrow p \Sigma^{0} K^{+}$reactions at the same excess energy $(\epsilon)$ [defined as $\epsilon=\sqrt{s}-m_{p}-m_{Y}-m_{K}$, with $m_{p}, m_{Y}$, and $m_{K}$ being the masses of proton, hyperon $(\mathrm{Y})$, and kaon, respectively, and $\sqrt{s}$ the invariant mass of the collision], is about $28_{-9}^{+6}$ for $\epsilon<13 \mathrm{MeV}$ [2]. This result is very intriguing because at higher beam energies this ratio is only about 2.5 [1]].

Assuming that the hyperon production proceeds solely due to the kaon $(K)$-exchange mechanism and that the final state interaction (FSI) effects among the outgoing particles are absent, $R$ is given essentially by the ratio of the squares of coupling constants at the vertices from which the $K^{+}$meson emerges $\left(g_{N \Lambda K}^{2} / g_{N \Sigma K}^{2}\right)$. The $\mathrm{SU}(6)$ prediction of this quantity is 27 [12] which would nearly explain the observed value of R. However, $\pi$-exchange mechanism is important for these reactions which taken together with the $K$ exchange process leads to a considerably lower value [2] $(\sim 3.6)$ for $R$. Of course, the $\Sigma^{0}$ production can be suppressed by the $\Sigma N \rightarrow \Lambda N$ conversion process in the $\Sigma-p$ final state interaction. However, there is no clear evidence in support of the fact that the whole of the observed enhancement is really due to the produced $\Sigma$ particle being converted to $\Lambda$ by the FSI effects.

Several theoretical studies have been performed to understand these data. These include the calculations of the Jülich group [13] and of Laget [14], which take into account both $\pi$ and $K$ exchange mechanisms and include final state interaction (FSI) effects within coupled 
channels approaches, and the resonance models [15] that consider the exchange of $\pi$ and other heavier mesons with excitations of intermediate baryonic resonant states which are coupled to kaon-hyperon channels. In yet another work [16], the near threshold hyperon production reported in Ref. 2] (for $\epsilon<13 \mathrm{MeV}$ ), have been analyzed within an effective Lagrangian model (ELM) which is developed [16, 17, 18, 19, 20] to investigate the particle production in nucleon-nucleon $(N N)$ collisions.

In the ELM, the initial interaction between two incoming nucleons is modeled by an effective Lagrangian which is based on the exchange of the $\pi, \rho, \omega$, and $\sigma$ mesons. The coupling constants at the nucleon-nucleon-meson vertices are determined by directly fitting the T-matrices of the nucleon-nucleon $(N N)$ scattering to the proton-proton and protonneutron scattering data in the relevant energy region. In these calculations the pseudovector (PV) coupling is used for the nucleon-nucleon-pion vertex as it is consistent with the chiral symmetry requirement of the QCD [21] and also it leads to negligible contributions from the negative energy states ("pair suppression phenomena") [22]. The particle production within the ELM proceeds via excitation of the relevant intermediate baryonic resonant states. To describe the near threshold data, the FSI effects in the final channel are included within the framework of the Watson-Migdal theory [23, 24]. ELA has been used so far to describe the $p p \rightarrow p p \pi^{0}, p p \rightarrow p n \pi^{+}$[17, 18], $p p \rightarrow p K^{+} Y$ [16, 19] as well as $p p \rightarrow p p e^{+} e^{-}$[20] reactions.

In Ref. [16], $N^{*}(1650), N^{*}(1710)$, and $N^{*}(1720)$ baryonic resonances were included as intermediate states in the ELM calculations of total cross sections of both $p p \rightarrow p \Lambda K^{+}$ $\left(\sigma_{t o t}^{p \Lambda K^{+}}\right)$and $p p \rightarrow p \Sigma^{0} K^{+}\left(\sigma_{\text {tot }}^{p \Sigma^{0} K^{+}}\right)$reactions. In that study, it was concluded that the contributions of the $N^{*}(1650)$ resonance state dominate both these reactions for $\epsilon$ values below $13 \mathrm{MeV}$. It was also noted that only with contributions of this resonance state included in both $\sigma_{t o t}^{p \Lambda K^{+}}$and $\sigma_{t o t}^{p \Sigma^{0} K^{+}}$, can the theory explain (within a factor of 2) the large values of $R$ in this very close-to-threshold energy regime.

Recently, the COSY-11 collaboration has published [3] data for both total cross sections for these reactions as well as their ratios in an extended energy regime (corresponding to $\epsilon$ values up to $60 \mathrm{MeV}$ ). It is observed that $R$ decreases strongly for $\epsilon$ between $10 \mathrm{MeV}-20$ $\mathrm{MeV}$. Beyond this the slope of $R$ is much smaller and the ratio slowly approaches to values observed at higher energies. Very recently, both COSY-11 and COSY-TOF collaborations have announced measurements of $p p \rightarrow p \Sigma^{+} K^{0}$ and $p p \rightarrow n \Sigma^{+} K^{+}$reactions [25, 26]. The analyzes of the data are in progress and the numerical values for the corresponding cross 
sections are likely to be available soon. Furthermore, there are also proposals to measure the associated hyperon production in pn collisions. Already the ANKE collaboration has reported 27] the estimated ratio of the cross sections for $p n \rightarrow n \Lambda K^{+}$and $p p \rightarrow p \Lambda K^{+}$ reactions at two near threshold beam energies.

The aim of this paper is to investigate how far can the trends of the data in the larger regime of near threshold energies be explained within the ELM. We would like to know if there are changes in the pattern of the relative contributions of various resonances to the total cross sections of the two reactions for $\epsilon$ values larger than $13 \mathrm{MeV}$. Furthermore, in view of the experimental activity on the $p p \rightarrow p \Sigma^{+} K^{0}$ and $p p \rightarrow n \Sigma^{+} K^{+}$reactions, it would be useful to give the predictions of our model for these channels. We also give result for the ratio of the $p p \rightarrow p \Lambda K^{+}$and $p n \rightarrow n \Lambda K^{+}$reactions at a near threshold beam energy.

In the present form of our effective Lagrangian model, the energy dependence of the cross section due to FSI is separated from that of the primary production amplitude and the total amplitude is written as [23]

$$
A_{f i}=M_{f i}\left(p p \rightarrow p Y K^{+}\right) \cdot T_{f f}
$$

where $M_{f i}\left(p p \rightarrow p Y K^{+}\right)$is the primary associated $Y K$ production amplitude, while $T_{f f}$ describes the rescattering among the final particles which goes to unity in the limit of no FSI. The Coulomb effects were included by using the modified formula for the effective range expansion of the phase-shift as discussed in [19]. This type of approach has been used extensively to describe the pion [28, 29], $\eta$-meson [30, 31, 32], associated hyperon [33], and $\phi$-meson [34] production in $p p$ collisions.

The amplitude $M_{f i}$ is determined by following the procedure described in Ref. [19]. The associated $K^{+} Y$ production proceeds via the excitation, propagation and decay of $N^{*}(1650)\left(\frac{1}{2}^{-}\right), N^{*}(1710)\left(\frac{1}{2}^{+}\right)$, and $N^{*}(1720)\left(\frac{3}{2}^{+}\right)$intermediate resonant states as below 2 $\mathrm{GeV}$ center of mass (c.m.) energy, only these resonance have significant decay branching ratios into the $K Y$ channels. Since all the three resonances can couple to the meson-nucleon channels mentioned earlier, we require the effective Lagrangians for all the four resonancenucleon-meson vertices corresponding to all the included resonances. One has the freedom of choosing either psuedoscalar (PS) and PV couplings for the $N^{*} N \pi$ and $N^{*} \Lambda Y$ vertices. In Ref. [19], $\sigma_{\text {tot }}^{p \Lambda K^{+}}$was calculated using both PS and PV couplings for these vertices. However, it was noted that the PS couplings for these vertices are clearly favored by the data. In this 
study we have used the PS couplings for these vertices. For all the details about the effective Lagrangians for these vertices and the determination of the coupling constants appearing therein, the propagators for the exchanged mesons and intermediate resonances and expressions for various amplitudes involved in $M_{f i}$, we refer to Ref. [19]. The amplitude $T_{f f}$ has been calculated by following the Jost function [23, 24] method using the Coulomb modified effective range expansion of the phase-shift in the same way as discussed in Refs. [16, 18, 19]. The required effective range and scattering length parameters were the same as those used in Refs. [16, 19] (set $\tilde{A}$ of Ref. [35]).

The factorization of the total amplitude into those of the primary production and FSI [Eq. (1)], enables one to pursue the diagrammatic approach for the meson production process within an effective Lagrangian model and investigate the role of various meson exchanges and resonances. At the same time it also allows the inclusion FSI effects among all the three outgoing particles. However, some difficulties inherent in the application of the WatsonMigdal method in calculations of the total amplitude for such reactions, should be kept in mind. Firstly, it has been argued in Refs. [36, 37] that although the energy dependence of the production process may be described correctly by Eq. (1) (particularly for the production of heavier mesons), its absolute magnitude could be uncertain because of the off-shell effects. Secondly, in Ref. [10] it is shown that the application of the Jost function method (with effective range approximation) for extracting the scattering length $\left(a_{S}\right)$ parameter for the $\Lambda-$ $p$ final state interaction from the $p p \rightarrow p \Lambda K^{+}$data, leads to larger values of $a_{S}$ as compared to those predicted by $Y N$ interaction models. Thirdly, the Watson-Migdal approximation has no scope for the inclusion of the coupled channels effects.

The off-shell effects can be included by multiplying the on-shell FSI amplitude with a form factor as is done in Refs. [13, 14, 16]. We have followed here exactly the same procedure as in Ref. [16]. It should be noted that in this method, both absolute magnitude as well as shapes of the FSI factor are affected by the off-shell corrections. This form factor approach can be improved by using the off-shell structure of some realistic $Y N$ interaction which may take care, to some extent, of the second criticism described above. However, it could imply going beyond the factorization approach of Eq. (1). A full solution of the problem clearly has to wait until a fully relativistic theory of the two-body $\rightarrow$ three-body reactions is developed where final state interactions between outgoing particles are dynamically incorporated in the production amplitude (perhaps by including diagrams with interaction lines between 
outgoing particles).

TABLE I: Resonance parameters, the branching ratios for their decay to various channels and the corresponding coupling constants obtained therefrom. Coupling constants at the $N^{*} N \omega$ vertices are obtained from the vector meson dominance hypothesis (see, e.g. Ref. [19]).

\begin{tabular}{|c|c|c|c|c|}
\hline Resonance & $\begin{array}{l}\text { Width } \\
(\mathrm{GeV})\end{array}$ & Decay channel & $\begin{array}{c}\text { Branching } \\
\text { ratio }\end{array}$ & $g$ \\
\hline \multirow[t]{6}{*}{$N^{*}(1710)$} & \multirow[t]{6}{*}{0.100} & $N \pi$ & 0.150 & 1.0414 \\
\hline & & $N \rho$ & 0.150 & 4.1421 \\
\hline & & $N \omega$ & & 1.2224 \\
\hline & & $N \sigma$ & 0.170 & 0.6737 \\
\hline & & $\Lambda K$ & 0.150 & 6.1155 \\
\hline & & $\Sigma K$ & $0.030^{a}$ & 7.4393 \\
\hline \multirow[t]{6}{*}{$N^{*}(1720)$} & \multirow[t]{6}{*}{0.150} & $N \pi$ & 0.150 & 0.1469 \\
\hline & & $N \rho$ & 0.700 & 19.483 \\
\hline & & $N \omega$ & & 16.766 \\
\hline & & $N \sigma$ & 0.120 & 1.5557 \\
\hline & & $\Lambda K$ & 0.080 & 1.0132 \\
\hline & & $\Sigma K$ & $0.060^{b}$ & 3.0651 \\
\hline \multirow[t]{6}{*}{$N^{*}(1650)$} & \multirow[t]{6}{*}{0.150} & $N \pi$ & 0.700 & 0.8096 \\
\hline & & $N \rho$ & 0.08 & 2.6163 \\
\hline & & $N \omega$ & & 1.8013 \\
\hline & & $N \sigma$ & 0.025 & 2.5032 \\
\hline & & $\Lambda K$ & 0.070 & 0.7658 \\
\hline & & $\Sigma K$ & & 0.4500 \\
\hline
\end{tabular}

${ }^{a}$ value suggested in $\tilde{\partial 0},\left[\frac{41}{4}\right] 0.07 \pm 0.06$

${ }^{b}$ value suggested in [38, 41] $0.09 \pm 0.03$

Both magnitude and sign of the coupling constant $g_{N_{1 / 2^{-}}^{*}} \Sigma K$ of the $N^{*}(1650) \Sigma K$ vertex, are uncertain. This is also, to some extent, true for those of the $N^{*}(1710) \Sigma K$ and $N^{*}(1720) \Sigma K$ vertices because the corresponding decay branching ratios are known only 
within a very broad range of values [38]. In Ref. [16], the magnitude of $g_{N_{1 / 2^{-}}^{*}} \Sigma K$ was taken to be 0.45 while its sign was assigned to be negative. These were determined in a preliminary investigation [16, 39] where fits were performed to the available data on the $\pi^{+} p \rightarrow \Sigma^{+} K^{+}$, $\pi^{-} p \rightarrow \Sigma^{0} K^{0}$, and $\pi^{-} p \rightarrow \Sigma^{-} K^{+}$reactions in an effective Lagrangian coupled channels approach [40]. Subsequently, in a more rigorous study [41], all the available data for transitions from $\pi N$ to five meson-baryon final states, $\pi N, \pi \pi N, \eta N, K \Lambda$, and $K \Sigma$ for center of mass energies ranging from threshold to $2 \mathrm{GeV}$, were fitted simultaneously. In these analyzes all the baryonic resonances with spin $\leq \frac{3}{2}$ (extended recently [42] to include also spin $\frac{5}{2}$ resonances) up to excitation energies of $2 \mathrm{GeV}$, are included as intermediate states. These studies indicate that the signs of the coupling constants for the vertices $N^{*}(1650) \Sigma K$, $N^{*}(1710) \Sigma K$, and $N^{*}(1720) \Sigma K$ could be identical (see, also, Ref. [43]). We found that a better fit to the hyperon production data is obtained with a positive sign for $g_{N_{1 / 2^{-}}^{*} \Sigma K}$ (the same as those of other two $N^{*} \Sigma K$ vertices). Thus while retaining the same value for its magnitude, we have used a positive sign for this coupling. Furthermore, the magnitudes of the coupling constants $g_{N_{1 / 2+}^{*}} \Sigma K$ and $g_{N^{*}} \Sigma_{2}$ are updated to values which are more in line with the recommendations of Refs. [38, 41, 43]. In Table I, we have shown the resonance properties and the branching ratios for their decay into various channels adopted for calculating the coupling constants of the corresponding vertices.

In Fig 1, we show the total cross sections for $p p \rightarrow p \Lambda K^{+}\left(\sigma_{t o t}^{p \Lambda K^{+}}\right)$and $p p \rightarrow p \Sigma K^{+}$ $\left(\sigma_{t o t}^{p \Sigma^{0} K^{+}}\right)$reactions as a function of the excess energy. We note that the calculations which are the coherent sum of all the resonance excitations and meson exchange processes as described earlier, are in good agreement with the data of the COSY-11 collaboration in the entire energy range. FSI effects are important for both the cases, even though the $p-\Sigma^{0}$ FSI is somewhat weaker than the $p-\Lambda$ one. The extended energy domain data of COSY-11 collaboration do indeed put a constraint on the $Y$-nucleon interaction as it was noted that the data in the entire energy range can be satisfactorily explained only with set $(\tilde{A})$ out of several sets of effective range and scattering length parameters for the hyperon-nucleon interaction given in Ref. [35].

The individual contributions of various nucleon resonances to $\sigma_{t o t}^{p \Lambda K^{+}}$and $\sigma_{\text {tot }}^{p \Sigma^{0} K^{+}}$are shown in Fig. 2. We note that in both the cases, the cross sections are dominated by the contributions from the $N^{*}(1650)$ resonance excitation for $\epsilon \leq 13 \mathrm{MeV}$ which was the energy range of the earlier COSY-11 data [2]. This is in agreement with the observations made 


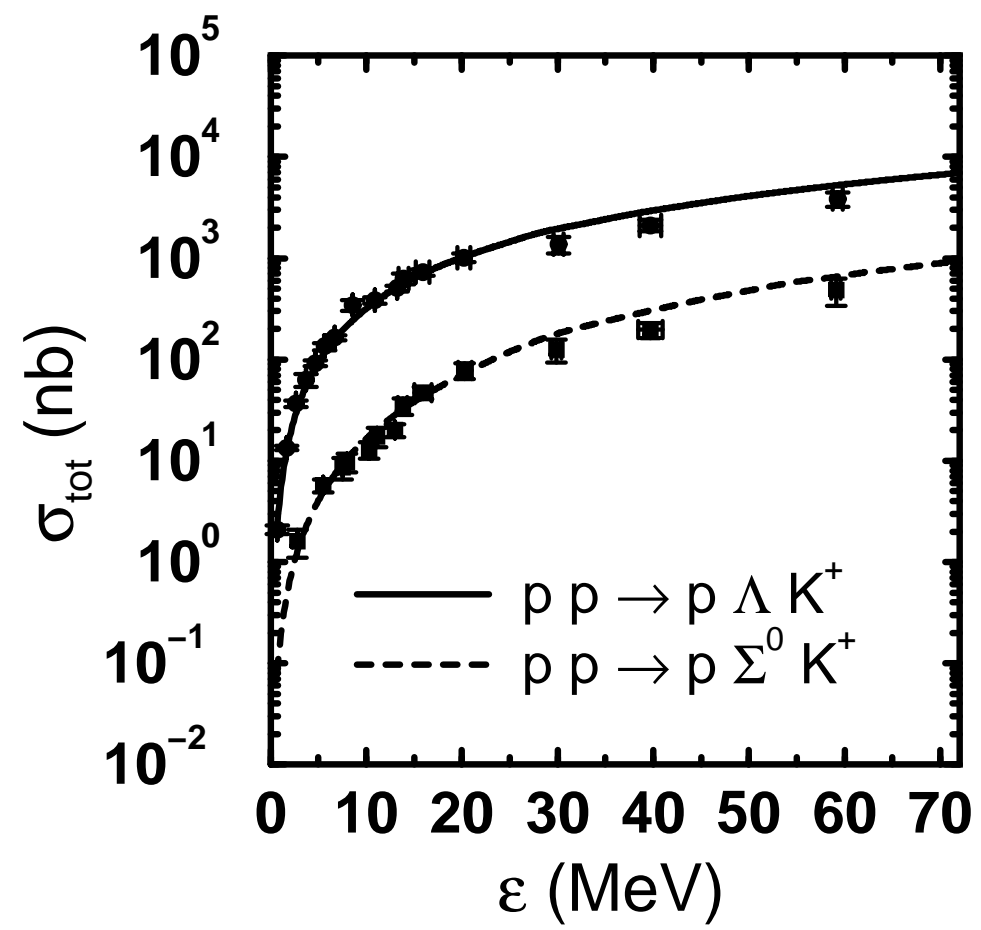

FIG. 1: Comparison of the calculated total cross sections for the $p p \rightarrow p \Lambda K^{+}$(solid line) and $p p \rightarrow p \Sigma^{0} K^{+}$(dashed line) reactions with the corresponding experimental data which are taken from Refs. [2, [3]

in Ref. [16]. Since $N^{*}(1650)$ is the lowest energy baryonic resonance which can decay to $Y K^{+}$channels, its dominance is to be expected in these reactions at very close-to-threshold beam energies. 44]. In this energy regime the relative dominance of various resonances is determined by the dynamics of the reaction where a difference of about $60 \mathrm{MeV}$ in excitation energies of $N^{*}(1650)$ and $N^{*}(1710)$ resonances plays a crucial role.

However, for $\epsilon$ values beyond $15 \mathrm{MeV}$, while the $p p \rightarrow p \Lambda K^{+}$reaction continues to be dominated by the $N^{*}(1650)$ excitation, the $p p \rightarrow p \Sigma^{0} K^{+}$reaction gets significant contributions also from higher mass resonances. In fact, $N^{*}(1720)$ resonance, dominates this channel for $\epsilon>30 \mathrm{MeV}$. Simple kinematics suggests that for a resonance inter- 


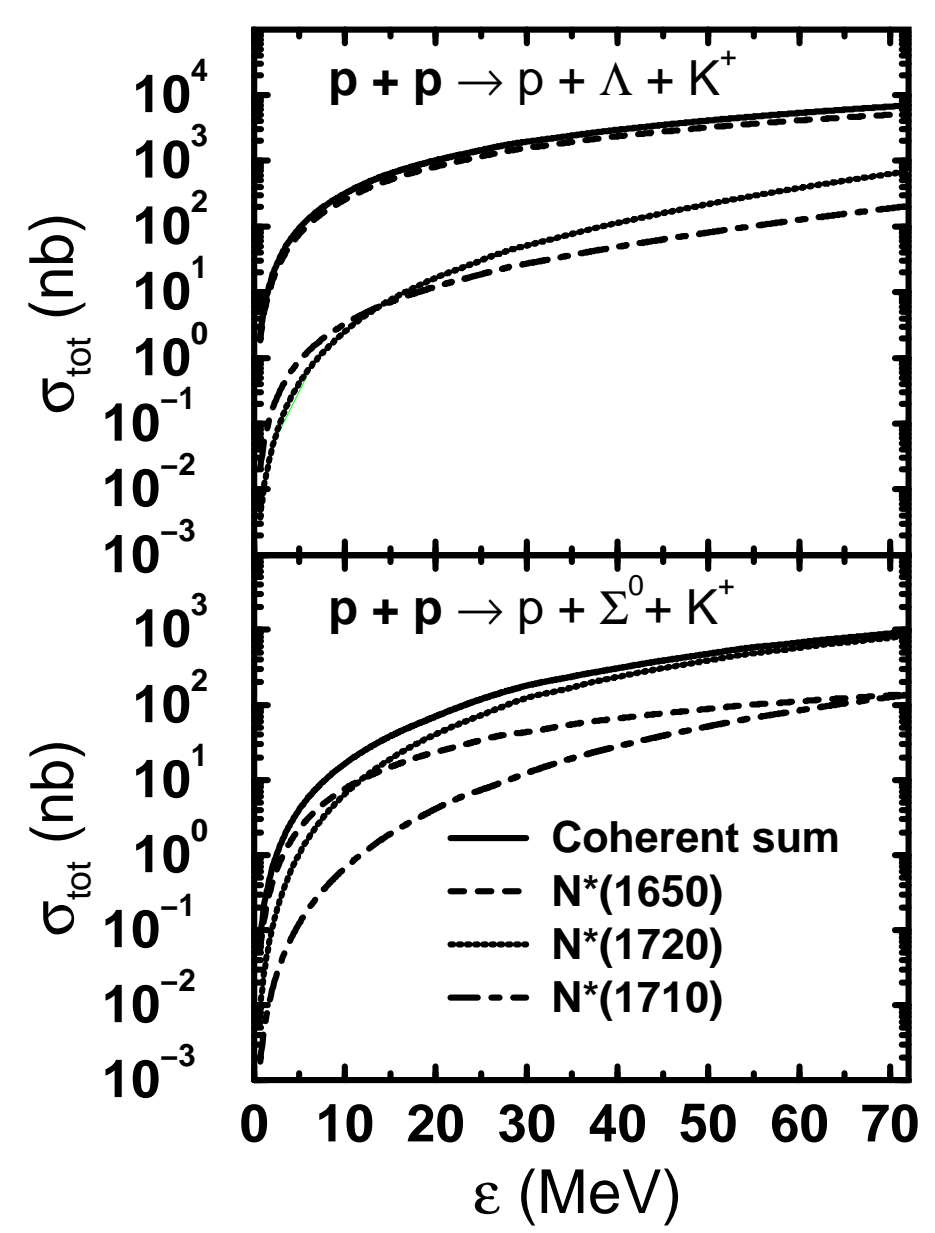

FIG. 2: Contributions of $N^{*}(1650)$ (dashed line), $N^{*}(1710)$ (dashed-dotted) and $N^{*}(1720)$ (dotted line) baryonic resonances to the total cross section for $p p \rightarrow p \Lambda K^{+}$(upper panel) and $p p \rightarrow p \Sigma^{0} K^{+}$ (lower panel) reactions. Their coherent sum is shown by solid lines.

mediate state to contribute significantly to the cross section, the values of $\epsilon$ should be $<Q\left[=m_{N^{*}}+\Gamma_{N^{*}} / 2-m_{Y}-m_{K}\right.$, where $m_{N^{*}}$ and $\Gamma_{N^{*}}$ are the mass and the width of the resonance, respectively]. For $N^{*}(1650)$, the values of $\mathrm{Q}$ are $38 \mathrm{MeV}$ and $115 \mathrm{MeV}$ for $p p \rightarrow p \Sigma^{0} K^{+}$and $p p \rightarrow p \Lambda K^{+}$reactions, respectively. Therefore, the range of $\epsilon$ in which this resonance should be contributing significantly is expected to be quite narrow in the case of the $p p \rightarrow p \Sigma^{0} K^{+}$reaction as compared to the $p p \rightarrow p \Lambda K^{+}$case. In this context, it would be worthwhile to note that in the recent Dalitz plot analyzes of data for the $p p \rightarrow p \Lambda K^{+}$ reaction by the COSY-TOF collaboration (see, eg., Refs. [26, 45]), it has been observed that even at the beam momentum of $2.85 \mathrm{GeV} / \mathrm{c}(\epsilon \approx 171 \mathrm{MeV})$, this reaction is domi- 
nated by the contributions from the $N^{*}(1650)$ resonance excitation. Similar analysis of the $p p \rightarrow p \Sigma^{0} K^{+}$reaction would be very instructive and useful.

Since in some of the earlier resonance model calculations the excitation of the $N^{*}(1650)$ resonance intermediate state was not included in the calculation of $\sigma_{t o t}^{p \Sigma^{0} K^{+}}$, we emphasize the importance of this resonance in describing the $p p \rightarrow p \Sigma^{0} K^{+}$at very close-to-threshold energies in Fig. 3. In this figure we we have shown $\sigma_{t o t}^{p \Sigma^{0} K^{+}}$(upper panel) and the ratio $(R)$ of $\sigma_{t o t}^{p \Lambda K^{+}}$and $\sigma_{t o t}^{p \Sigma^{0} K^{+}}$(lower panel) as a function of $\epsilon$, with and without including the contributions of $N^{*}(1650)$ intermediate resonance state in $\sigma_{t o t}^{p \Sigma^{0} K^{+}}$. It is clear that without contributions of the $N^{*}(1650)$ resonance, $\sigma_{t o t}^{p \Sigma^{0} K^{+}}$is underpredicted roughly by an order of magnitude for $\epsilon$ values very close to the production threshold. As $\epsilon$ increases the contributions of this resonance becomes lesser and lesser important.

This trend of $\sigma_{\text {tot }}^{p \Sigma^{0} K^{+}}$is directly reflected in the behavior of $R$. In the lower panel of Fig. 3 , we have shown our results for $R$ with and without considering $N^{*}(1650)$ excitation in the calculations of $\sigma_{t o t}^{p \Sigma^{0} K^{+}}$. In both the results shown here, we have used full $\sigma_{t o t}^{p \Lambda K^{+}}$(including contributions of all the three resonances) in the calculations of $R$. It is clear that for $\epsilon<20$ $\mathrm{MeV}, R$ is overpredicted by factors ranging from 8 to 4 if $N^{*}(1650)$ contributions are not included in $\sigma_{t o t}^{p \Sigma^{0} K^{+}}$. Due to reducing significance of these contributions to $\sigma_{t o t}^{p \Sigma^{0} K^{+}}$with increasing $\epsilon$, the difference between the two calculations become smaller for bigger values of $\epsilon$. At larger beam energies the smaller values of $R$, therefore, are due to the difference in the resonance contribution pattern. In this regime, the $N^{*}(1720)$ and $N^{*}(1710)$ resonances play a different role for both these reactions. Therefore, this is yet another example of the sensitivity of the COSY-11 data to the details of the dynamics of resonance contributions. It should also be mentioned here that that our full calculations are able to explain the trend of the energy dependence of $R$ rather well.

Some authors [13, 14] have used a different picture to understand these data. In the calculations reported by the Jülich group [13], the initial $N N$ collisions are modeled in terms of both $\pi$ and $K$ exchange processes and the FSI effects are included in a coupled channels approach. They show that while $\Lambda K^{+}$production channel is dominated by the $K$ exchange mechanism, both $\pi$ and $K$ exchange diagrams contribute with almost equal strength to the $\Sigma^{0} K^{+}$channel. With the assumption of a destructive interference between the two amplitudes, this model is able to explain large values of $R$ for $\epsilon$ corresponding to beam energies very close to the production threshold. In calculations reported in Ref. [14] too 


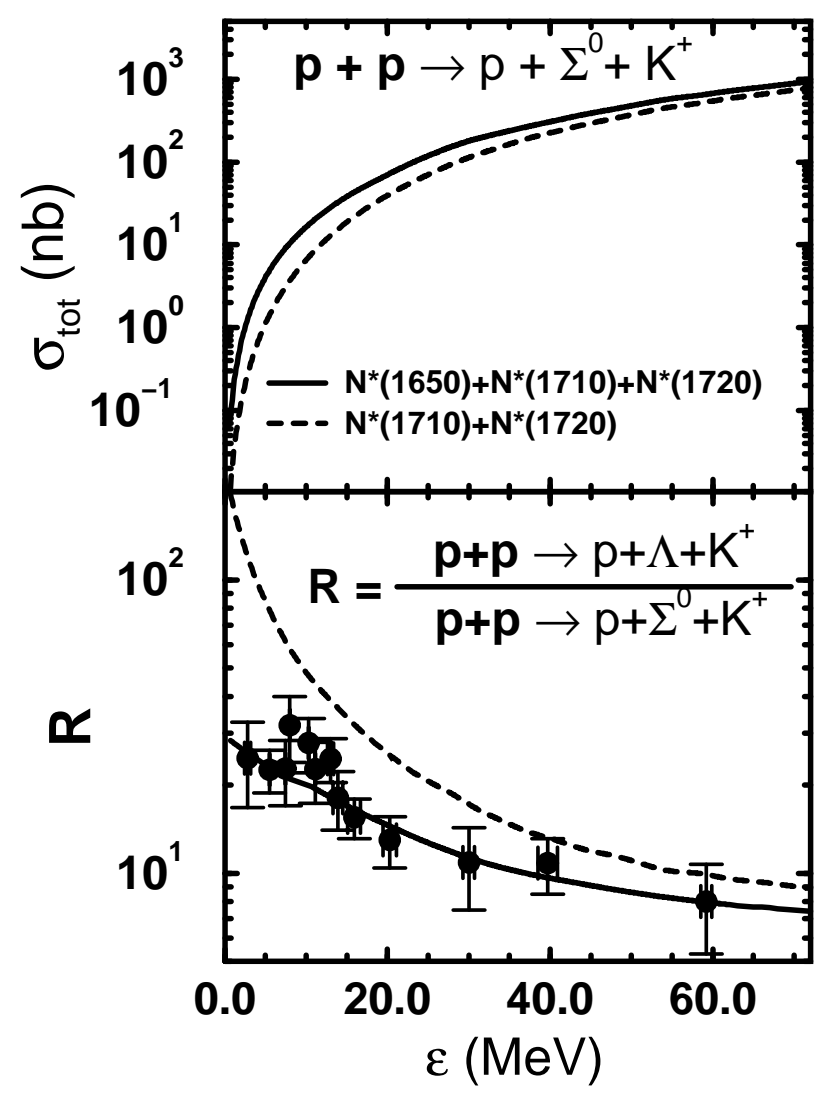

FIG. 3: Role of $N^{*}(1650)$ resonance in the total cross section for the $p p \rightarrow p \Sigma^{0} K^{+}$reaction (upper panel) and in the ratio $R$ of the $p p \rightarrow p \Lambda K^{+}$and $p p \rightarrow p \Sigma^{0} K^{+}$reactions (lower panel) as a function of excess energy. The solid lines show the results of full calculations while the dashed lines are the ones obtained without including the $N^{*}(1650)$ resonance contributions in the cross sections for the $p p \rightarrow p \Sigma^{0} K^{+}$reaction. In both the cases, full $\sigma_{t o t}^{p \Lambda K^{+}}$including contributions from all the three resonant states, have been used in the calculations o $R$. The data are from Refs. 2, 3].

the relative sign of $K$ and $\pi$ exchange terms is chosen solely by the criteria of reproducing the experimental data, although in this work the theory has been applied to describe a wider range of data including the polarization transfer results of the DISTO experiment 46] and the missing mass distribution obtained in the inclusive $K^{+}$production measurements performed at SATURNE [4]]. However, the observed energy dependence of $R$ is yet to be explained within both these calculations. More work on these models (e.g, including the heavy vector meson exchanges [48] and finding a way to fixed the relative sign of the $\pi$ and $K$ exchange amplitudes) would be worth pursuing as it is an interesting alternative approach 


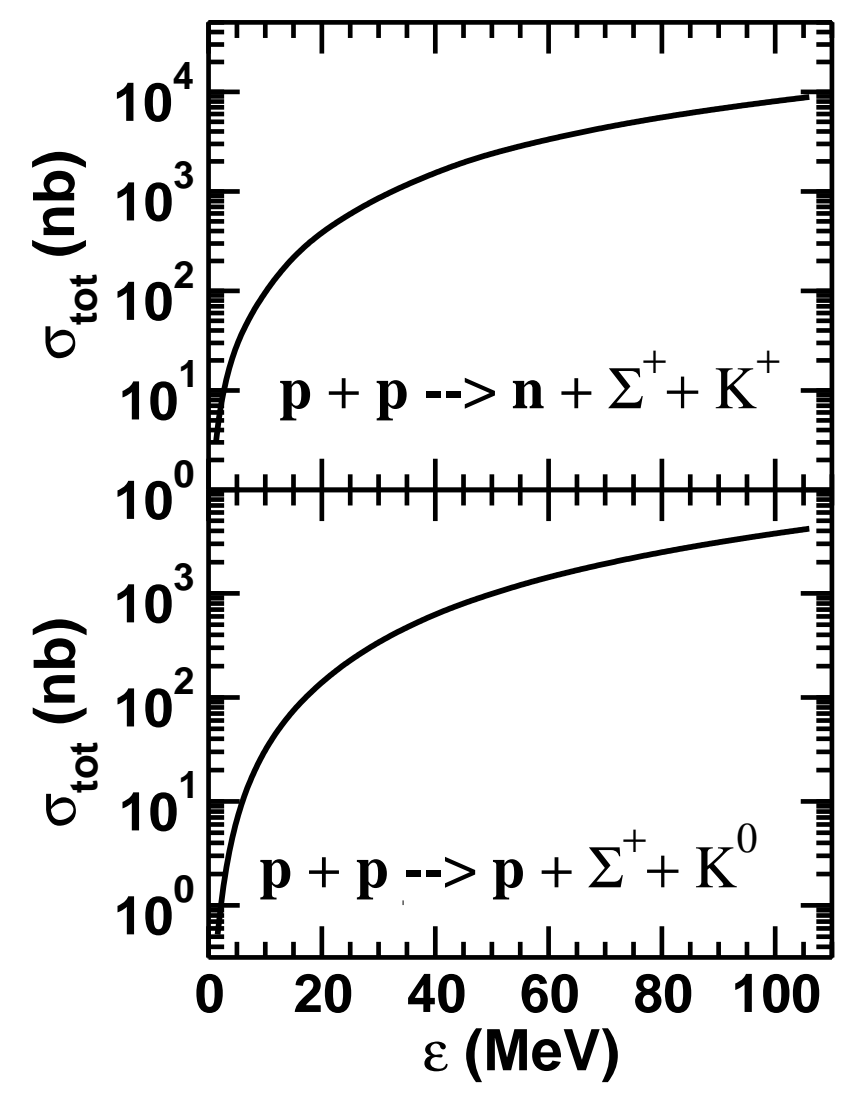

FIG. 4: Total cross sections for the $p p \rightarrow n \Sigma^{+} K^{+}$and $p p \rightarrow p \Sigma^{+} K^{0}$ reaction as a function of the excess energy.

to the resonance excitation picture.

Recently, both COSY-11 [25] and COSY-TOF [26] collaborations have announced measurements of the total cross sections for the $p p \rightarrow n \Sigma^{+} K^{+}$and $p p \rightarrow p \Sigma^{+} K^{0}$ reactions. The interest in the $\Sigma^{+}$production channel stems also from the fact that it provides a sensitive tool to search for a possible penta quark state [5]. The total cross section data are currently being analyzed. We show in Fig. 4, the prediction of our model for these reactions. The isospin factors for different channels are shown in Table II. Except for slightly different hyperon and strange meson masses, all other parameters were taken to be the same as those described above. It is hoped that soon there data would be available to check our predictions.

We also calculated the total cross section for the $p n \rightarrow n \Lambda K^{+}$reaction $\left(\sigma_{t o t}^{n \Lambda K^{+}}\right)$. Apart from the different isospin factors, all the parameters were the same in calculations of both the reactions. The ratio of $\sigma_{t o t}^{n \Lambda K^{+}}$and $\sigma_{t o t}^{p \Lambda K^{+}}$is found to be 2.4 at the beam energy of 
TABLE II: Isospin factors for various diagrams, Isovector corresponds to $\pi$ and $\rho$ exchange graphs while isoscalar to $\omega$ and $\sigma$ ones

\begin{tabular}{|ccc|}
\hline \hline graph & isovector & isoscaler \\
\hline direct & $p n \rightarrow n \Lambda K^{+}$ & \\
exchange & -1.0 & -1.0 \\
& 2.0 & 0.0 \\
direct & $p p \rightarrow p \Sigma^{+} K^{0}$ & \\
exchange & $-\sqrt{2}$ & $-\sqrt{2}$ \\
& $-\sqrt{2}$ & $-\sqrt{2}$ \\
direct & $p p \rightarrow n \Sigma^{+} K^{+}$ & 0 \\
exchange & $2 \sqrt{2}$ & 0 \\
\hline \hline
\end{tabular}

$1.83 \mathrm{GeV}$. This compares well with the value extracted in Ref. [27] from the analysis of the inclusive $K^{+}$production data.

In summary, we have studied the $p p \rightarrow p \Lambda K^{+}, p n \rightarrow n \Lambda K^{+}, p p \rightarrow p \Sigma^{0} K^{+}, p p \rightarrow$ $n \Sigma^{+} K^{+}$, and $p p \rightarrow p \Sigma^{+} K^{0}$ reactions within an effective Lagrangian model in an extended regime of near threshold beam energies. The reactions proceed via the excitation of the $N^{*}(1650), N^{*}(1710)$, and $N^{*}(1720)$ intermediate baryonic resonant states. We confirm that the $N^{*}(1650)$ resonant state contributes predominantly to the cross sections of all these reactions at very close-to-threshold beam energies. Therefore, in this energy regime, hyperon production reactions in nucleon-nucleon collisions provide an interesting tool for investigating the properties of this negative parity spin- $\frac{1}{2}$, isospin- $\frac{1}{2}$ resonance much the same way way as the $\eta$ meson production probes the lower energy $N^{*}(1535)$ which has the same parity, spin and isospin.

The inclusion of the contributions of the $N^{*}(1650)$ resonance in the cross sections of both $p p \rightarrow p \Sigma^{0} K^{+}$and $p p \rightarrow p \Lambda K^{+}$eactions, is essential to explain the experimentally observed large values of their ratios at very small values of the excess energies (or beam energies). This is also necessary for explaining the beam energy dependence of this ratio. This result of course assumes that the final state interaction effects in the exit channel can be accounted 
for by the Watson-Migdal theory.

At larger near threshold beam energies (for excess energies between 20-60 MeV), while $p p \rightarrow p \Lambda K^{+}$reaction continues to be dominated by the $N^{*}(1650)$ resonance, the $p p \rightarrow$ $p \Sigma^{0} K^{+}$reaction gets significant contributions also from the $N^{*}(1720)$ and $N^{*}(1710)$ resonances. A Dalitz plot analysis of the data for this reaction would be very instructive. We also give our predictions for the cross sections of the $p p \rightarrow p \Sigma^{+} K^{0}$ and $p p \rightarrow n \Sigma^{+} K^{+}$reactions. It is hoped that data for these channels will soon be available so that our predictions can be tested. The calculated ratio of the total cross sections for $p p \rightarrow p \Lambda K^{+}$and $p n \rightarrow n \Lambda K^{+}$ reactions at the beam energy of $1.83 \mathrm{GeV}$ is found to be 2.4 which is very close to the value extracted in an analysis of the inclusive $K^{+}$production data.

This work was done when the author was visiting Department of Radiation Sciences of the Uppsala University, Sweden. His stay in Uppsala was supported by the WennerGren Foundation, Stockholm. He would like to thank Anders Ingemarsson for inviting him to Uppsala and for several helpful discussions. Useful conversations with Bo Höistad and Göran Fäldt are also gratefully acknowledged.

[1] J. Balewski et al., Phys. Lett. B 420, 211 (1998).

[2] S. Sewerin et al., Phys. Rev. Lett. 83, 682 (1999).

[3] P. Kowina et. al., Eur. Phys. J. A22, 293 (2004).

[4] R. Bilger et al., Phys. Lett. B 420, 217 (1998).

[5] M. Abdel-Bary et al., Phys. Lett. B595, 127 (2004).

[6] T.E.O Ericson and W. Weise, Pions and Nuclei, Clarendon, Oxford, 1988; V. Bernard, N. Kaiser, and Ulf-G. Meissner, Int. J. Mod. Phys. E4, 193 (1995); C. Hahnhart, Phys. Rep. 397, 155 (2004).

[7] M. Alberg, Prog. Part. Nucl. Phys. 36, 217 (1996).

[8] P. Moskal, M. Wolke, A. Khoukaz, and W. Oelert, Prog. Part. Nucl. Phys. 49, 1 (2002).

[9] N. Kelkar and B.K. Jain, Int. J. Mod. Phys. E9, 431 (2000).

[10] A. Gasparyan, J. Haidenbauer, and C. Hanhart, Phys. Rev. C 72, 034006 (2005); A. Gasparyan, J. Haidenbauer, C. Hanhart, and J. Speth, Phys. Rev. C 69, 034006 (2004).

[11] Landolt-Börnstein: Numerical Data and Functional Relationships in Science and Technology, 
New Series, edited by H. schopper, I/12 (Springer, Berline, 1988).

[12] J. J. De Swart, Rev. Mod. Phys. 35, 916 (1963); C. B. Dover and A. Gal, Prog. Part. Nucl. Phys. 12, 171 (1984).

[13] A.M. Gasparyan, J. Haidenbauer, C. Hanhart, L. Kondratyuk, and J. Speth, Phys. Lett. B 480, $273(2000)$.

[14] J. M. Laget, Nucl. Phys. A691, 11c (2001); ibid., Phys. Lett. B359, 24 (1991).

[15] K. Tsushima, A. Sibirtsev and A.W. Thomas, Phys. Lett. B390, 29 (1997); ibid., Phys. Rev. C 59, 369 (1999), Erratum, C61, 029903 (2000).

[16] R. Shyam, G. Penner, and U. Mosel, Phys. Rev. C 63, 022202(R) (2001).

[17] A. Engel, R. Shyam, U. Mosel and A.K. Dutt-Majumdaer, Nucl. Phys.A 603, 387 (1996).

[18] R. Shyam and U. Mosel, Phys. Lett. B 425, 1 (1998)

[19] R. Shyam, Phys. Rev. C 60, 055213 (1999).

[20] R. Shyam and U. Mosel, Phys. Rev. C 67, 065202 (2003).

[21] S. Weinberg, Phys. Rev. 166, 1568 (1996).

[22] R. Machleidt, K. Holinde and Ch. Elster, Phys. Rep. 149, 1 (1987).

[23] M.L. Goldberger and K.M. Watson, Collision Theory, Wiley, New York, 1969, pp 549.

[24] J. Gillespie, Final-State Interaction, Ed. K. M. Watson, Holden-Day Adv. Phys. Monographs, Holden-Day, San Francisco, 1964.

[25] T. Rozek, et al., Int. J. Mod. Phys. A20, 680 (2005).

[26] M. Bücher, Nucl. Phys. A754, 231c (2005).

[27] M. Bücher et al., Eur. Phys. J. A 22, 301 (2004).

[28] J. Dubach, W.M. Kloet, and R.R. Silbar, Phys. Rev. C 33, 373 (1986).

[29] V. Bernard, N. Kaiser, and Ulf-G. Meissner, Eur. Phys. J. A 4, 259 (1999).

[30] A. Moalem, E. Gedalin, L. Razdolskaja, and Z. Shorer, Nucl. Phys. A 600, 455 (1996).

[31] B.L. Druzhinin, A.E. Kudryavtsev, and V.E. Tarasev, Z. Phys. A 359, 205 (1997).

[32] A. Deloff, Phys. Rev. C 69, 035206 (2004).

[33] A. Sibirtsev, K. Tsushima, W. Cassing, and A.W. Thomas, arXiv:ucl-th/0004022.

[34] A.I. Titov, B. Kämpfer, and B.L. Reznik, Eur. Phys. J. A 7, 543 (2000).

[35] R. Reuber, K. Holinde, and J. Speth, Nucl. Phys. A570, 543 (1994).

[36] C. Hanhart and K. Nakayama, Phys. Lett. B454, 176 (1999).

[37] V. Baru, A.M. Gasparyan, J. Haidenbauer, A.E. Kudryavtsev, and J. Speth, Phys. Atom. 
Nucl. 64, 579 (2001) [Yad. Fiz. 64, 633 (2001)].

[38] S. Eidelman et al., Phys. Lett. B592, 1 (2004).

[39] A. Waluyo, C. Benhold, H. Haberzettl, G. Penner, U. Mosel, and T. Mart, arXiv:nucl-th/0008023.

[40] T. Feuster and U. Mosel, Phys. Rev. C 58, 457 (1998) ibid. 59, 460 (1999).

[41] G. Penner and U. Mosel, Phys. Rev. C 66, 055211 (2002); 66, 055212 (2002).

[42] V. Shklyar, H. Lenske, and U. Mosel, Phys. Rev. C 72, 015210 (2005).

[43] G. Penner, Ph.D. thesis (in English), Universität Giessen, 2002.

[44] G. Fäldt and C. Wilkin, Z. Phys. A 357, 241 (1997).

[45] W. K. Eyrich, Prog. Part. Nucl. Phys. 50, 547 (2003).

[46] F. Balestra et al., Phys. Rev. Lett. 83, 1534 (1999).

[47] R. Siebert et al., Nucl. Phys. A567 (1994)819.

[48] N. Kaiser, Eur. Phys. J. A 5, 105 (1999). 